\title{
Chiral and deconfinement transitions in strong coupling lattice QCD
}

\section{Kohtaroh Miura*}

INFN Laboratori Nazionali di Frascati, I-00044, Frascati (RM), Italy

E-mail: Kohtaroh.Miuraddnf.infn.it

\section{Takashi Z. Nakano}

Department of Physics, Faculty of Science, Kyoto University, Kyoto 606-8502, Japan

Yukawa Institute for Theoretical Physics, Kyoto University, Kyoto 606-8502, Japan

\section{Akira Ohnishi}

Yukawa Institute for Theoretical Physics, Kyoto University, Kyoto 606-8502, Japan

\section{Noboru Kawamoto}

Deparment of Physics, Faculty of Science, Hokkaido University, Sapporo 060-0810, Hokkaido,

Japan

\begin{abstract}
We investigate the QCD phase diagram based on the strong coupling expansion of the lattice QCD with one species of the staggered fermions at finite temperature $(T)$ and chemical potential $(\mu)$. We analytically derive an effective potential including both chiral and deconfinement $\left(Z_{N_{c}}\right)$ dynamics with finite coupling effects in mean-field approximations. We focus on Polyakov loop properties in whole $T-\mu$ plane, and study relations between the chrial and deconfinement crossovers. At a fixed large $\mu$, sequencial rapid variations of the Polyakov loop are observed with increasing $T$. It is natural to interprete them as the "chiral induced" and " $Z_{N_{c}}$ induced" deconfinements crossovers.
\end{abstract}

The XXVIII International Symposium on Lattice Field Theory, Lattice2010

June 14-19, 2010

Villasimius, Italy

\footnotetext{
* Speaker.
} 


\section{Introduction}

The strongly interacting non-Abelian gauge theory is a central key-concept in the high energy physics. One of the most important properties is the asymptotic freedom, which indicates a phase transition (or crossover) from hadron phase to a deconfined matter -Quark-Gluon Plasma (QGP)in Quantum Chromodynamics (QCD) when temperature $(T)$ and/or quark chemical potential $(\mu)$ exceed a typical energy scale of QCD $\left(\Lambda_{\mathrm{QCD}}\right)$. To investigate the QGP and this phase transition is a physics goal in the LHC-ALICE experiment, and indispensable to understand the astrophysical systems, such as the early universe and the neutron stars.

The phase transition (or crossover) from the hadron to the QGP can be described in terms of two kinds of (approximate) symmetries, the $\mathrm{SU}\left(N_{f}\right) \times \mathrm{SU}\left(N_{f}\right)$ chiral symmetry and the center $Z_{N_{c}}$ symmetries ( $N_{c}$ and $N_{f}$ represents the number of colors and flavors). The finite value of the Polyakov loop indicates a sort of deconfinement in the sense that an excitation energy of heavy quark is finite. The finite value of the chiral condensate at vanishing current quark mass indicates the hadron phase in the sense that hadrons are massive (the pion is massless) and relevant degrees of freedom. The Polyakov loop and the chiral condensate breaks the $Z_{N_{c}}$ and the chiral symmetries, respectively, and are typical observables in the lattice QCD. The lattice QCD based investigation for the phase structure of their symmetries i.e. the $Q C D$ Phase Diagram in $T-\mu$ plane is one of the most important subjects in the high energy physics.

The Lattice QCD Monte-Carlo simulations (LQCD-MC) indicate that chiral and deconfinement $\left(Z_{N_{c}}\right)$ crossovers simultaneously happen around, $T_{c}=\simeq 160-190 \mathrm{MeV}$ at $\mu=0$ [四]. The coincidence of " $Z_{N_{c}}$ and chiral" is the non-trivial observation, and the relation between their crossovers is a modern interest on the QCD phase diagram. The important sampling method in LQCD-MCs suffers from the sign problem of the quark determinant with finite $\mu$, and the LQCD-MC does not work well at finite $\mu$. It is required to shed light on the large $\mu$ region of the QCD phase diagram beyond the sign problem.

In this proceedings, we investigate QCD phase diagram in the whole range of $T-\mu$ plane by using the Strong Coupling Expansion in Lattice QCD (SC-LQCD) [[]] with one species of (unrooted) staggered fermion. The analytic investigation based on SC-LQCD can be informative for finite $\mu$, and there are many recent developments [ $[3]-Q]$. We take account of next-to-leading order (NLO, $\mathscr{O}\left(1 / g^{2}\right)$ ) terms in the strong coupling expansion. In addition, we consider the leading pure gluonic contributions for Polyakov loops. We concentrate on the leading order of the large dimensional $(1 / d)$ expansion [10] for simplicity. In these setups, we focus on the relation between the $U_{\chi}(1)$ chiral and $Z_{N_{c}}$ deconfinement dynamics in the phase diagram.

\section{Effective Potential}

We start from the lattice QCD partition function with one species of staggered fermion $(\chi)$ with a quark mass $\left(m_{0}\right)$. Gluons are represented by link variables $\left(U_{v, x}\right)$,

$$
Z=\int \mathscr{D}\left[\chi, \bar{\chi}, U_{v}\right] \exp \left[-\sum_{v, x} \frac{\eta_{v, x} \bar{\chi}_{x} U_{v, x} \chi_{x+\hat{v}}-\eta_{v, x}^{-1}(\text { h.c. })}{2}-\sum_{x} m_{0}(\bar{\chi} \chi)_{x}+\sum_{P} \frac{U_{P}+U_{P}^{\dagger}}{g^{2}}\right] .
$$


where, $U_{P=\mu v, x}=\operatorname{tr}_{c}\left[U_{\mu, x} U_{v, x+\hat{\mu}} U_{\mu, x+\hat{v}}^{\dagger} U_{v, x}^{\dagger}\right]$, and $\left(\eta_{0, x}, \eta_{j, x}\right)=\left(e^{\mu},(-1)^{x_{0}+\cdots+x_{j-1}}\right)$. By using a $\gamma_{5^{-}}$ related factor $\varepsilon_{x}=(-1)^{x_{0}+\cdots+x_{d}}$, a staggered chiral transformation is defined as $\chi_{x} \rightarrow e^{i \theta \varepsilon_{x}} \chi_{x}$ [[]]. The lattice action is invariant under this $U_{\chi}(1)$ chiral transformation in the chiral limit $m_{0} \rightarrow 0$. In the following, we use the lattice unit $a=1$, and we investigate the color $\mathrm{SU}\left(N_{c}=3\right)$ case in $3+1$ dimensions $(d=3)$.

We perform strong coupling expansions, and take account of the next-to-leading order (NLO) effects. To manipulate temperature effects, we firstly evaluate the spatial gluon degrees of freedom by utilizing the group integral formula, $\int d U_{j x} U_{j x_{a}^{b}}^{b} U_{j x_{c}}^{\dagger}=\delta_{a}^{d} \delta_{c}^{b} / N_{c}$, which leads to sums of color singlet (hadronic) composites. The spatial link integral leaves an isotropic sum over spatial directions, and the energy per bond is assumed to be proportional to $1 / d$ in order to keep the action finite at large spatial dimension $d[\mathbb{1 0}]$. In this normalization scheme, the quark field scales as $d^{-1 / 4}$. We concentrate on the leading order $1 / d$ terms, which correspond to the minimum quark number configurations for a given plaquette geometry. Within these approximations, we obtain the following hadronic composites and effective couplings,

$$
\begin{aligned}
& \left(\beta_{\tau}, \beta_{s}\right)=\left(\frac{d}{N_{c}^{2} g^{2}}, \frac{d(d-1)}{8 N_{c}^{4} g^{2}}\right)=\left(\frac{d}{2 N_{c}^{3}} \beta, \frac{d(d-1)}{16 N_{c}^{5}} \beta\right), \\
& \left(M_{x}, V_{x}^{+}(\mu), V_{x}^{-}(\mu)\right)=\left(\bar{\chi}_{x} \chi_{x}, e^{\mu} \bar{\chi}_{x} U_{0, x} \chi_{x+\hat{0}}, e^{-\mu} \bar{\chi}_{x+\hat{0}} U_{0, x}^{\dagger} \chi_{x}\right),
\end{aligned}
$$

where the lattice coupling $\beta=2 N_{c} / g^{2}$ is the unique parameter in the current theory. The left panel of Fig. $\square$ provides a schematic description of composites. In addition to them, we extract the leading pure gluonic effect to Polyakov loops $\left(L_{p, \mathbf{x}}=\prod_{\tau} U_{0, x}\right.$, the right hand side of Fig. 四), which is necessary to investigate a deconfinement dynamics. These setups give us the simplest framework to investigate both $U_{\chi}(1)$ chiral and $Z_{N_{c}}$ deconfinement transitions (or crossovers) in the SC-LQCD with finite coupling effects. Recent our study indicates that the next-to-next-to leading order effects with quarks has just a small correction to the phase diagram, while there is discrepancy between SCLQCD and the Lattice QCD Monte-Carlo simulations (LQCD-MC) for the $\beta=2 N_{c} / g^{2}$ evolutions of the critical temperature at zero chemical potential, $\left(T_{c, \mu=0}(\beta)\right)[[]]$. Due to the pure gluonic Polyakov loops, the $T_{c, \mu=0}(\beta)$ is found to become closer to the lattice Monte-Carlo (LQCD-MC) simulations around $\beta=4.0$ at $\mu=0$ [Q] ]. We anticipate that the pure gluonic Polyakov loops could have an essential contribution at "finite $\mu$ ", which will be investigated here.

To integrate the quark degrees of freedom $(\chi, \bar{\chi})$, we introduce four kinds of mean fields [ $[\mathbb{}]$, which are summarized in Table $\mathbb{W}$. The mean field $\sigma$ represents the chiral condensate, which leads to the dynamical mass shift, $m_{0} \rightarrow m_{q}=m_{0}+\left(d /\left(2 N_{c}\right)\right) \sigma$ in the strong coupling limit It is remarkable that the NLO effects are expressed as modifications of quark mass and chemical potential, $\left(m_{q}, \mu\right) \rightarrow\left(\tilde{m}_{q}, \tilde{\mu}\right)$ with the quark wave function renormalization factor, $\sqrt{Z_{+} Z_{-}}$, and those are governed by the unique lattice parameter $\beta[\square]$,

$$
\tilde{m}_{q}=\frac{1}{\sqrt{Z_{+} Z_{-}}}\left(m_{0}+\left(\frac{d}{2 N_{c}}+2 \beta_{s} \varphi_{s}\right) \sigma\right), \quad \tilde{\mu}=\mu-\log \sqrt{\frac{Z_{+}}{Z_{-}}},
$$

where, $Z_{ \pm}=1+\beta\left(\varphi_{\tau} \pm \omega_{\tau}\right)$.

This NLO formulation which is invented in our previous work $[\mathbb{}[]$ allows us to evaluate the remnant degrees of freedom, quarks $(\chi, \bar{\chi})$ and the temporal link variable $U_{0}$, in the same way to 
the strong coupling limit case [ए], [2]]. It is convenient to take a static and diagonalized gauge (called the Polyakov gauge) for temporal link variables with respect for the periodicity [प]], $U_{0, \mathbf{x}}=$ $\operatorname{diag}\left\{e^{i \theta_{\mathbf{x}}^{1} / N_{\tau}}, \cdots, e^{i \theta_{\mathbf{x}}^{N_{c}} / N_{\tau}}\right\}$. In this gauge, the Polyakov loop reduces to a simple expression, $L_{p, \mathbf{x}} \rightarrow$ $\sum_{a=1}^{N_{c}} e^{i \theta_{\mathbf{x}}^{a}} \equiv N_{c} l_{p, \mathbf{x}}$, and the Haar measure is also expressed in terms of the Polyakov loops,

$$
\begin{gathered}
\int d U_{0, \mathbf{x}} \rightarrow \int_{0}^{1} d\left[l_{p, \mathbf{x}}, \bar{l}_{p, \mathbf{x}}\right] 27 \mathscr{M}_{\text {Haar }}\left(l_{p, \mathbf{x}}, \bar{l}_{p, \mathbf{x}}\right) \\
\mathscr{M}_{\text {Haar }}\left(l_{p, \mathbf{x}}, \bar{l}_{p, \mathbf{x}}\right)=1-6\left(\bar{l}_{p, \mathbf{x}} l_{p, \mathbf{x}}\right)-3\left(\bar{l}_{p, \mathbf{x}} l_{p, \mathbf{x}}\right)^{2}+4\left(l_{p, \mathbf{x}}^{N_{c}}+\bar{l}_{p, \mathbf{x}}^{N_{c}}\right) .
\end{gathered}
$$

The Haar measure effects are responsible for the deconfinement dynamics. The quark determinant includes the quark propagation wrapping the temporal direction with $U_{0}$, and leads to the quark driven Polyakov loops, which is characterized by,

$$
\begin{aligned}
& \mathscr{D}_{q}(T, \mu) \equiv 1+N_{c}\left(l_{p, \mathbf{x}} e^{-\left(E_{q}-\tilde{\mu}\right) / T}+\bar{l}_{p, \mathbf{x}} e^{-2\left(E_{q}-\tilde{\mu}\right) / T}\right)+e^{-N_{c}\left(E_{q}-\tilde{\mu}\right) / T} \\
& \mathscr{D}_{\bar{q}}(T, \mu) \equiv 1+N_{c}\left(\bar{l}_{p, \mathbf{x}} e^{-\left(E_{q}+\tilde{\mu}\right) / T}+l_{p, \mathbf{x}} e^{-2\left(E_{q}+\tilde{\mu}\right) / T}\right)+e^{-N_{c}\left(E_{q}+\tilde{\mu}\right) / T}
\end{aligned}
$$

Here, $E_{q}\left(\tilde{m}_{q}(\sigma)\right)=\sinh ^{-1}\left[\tilde{m}_{q}(\sigma)\right]$ corresponds to the quark excitation energy. The Polyakov loop $l_{p}$ couples to a Boltzmann factor $e^{-\left(E_{q}-\tilde{\mu}\right) / T}$, and determines quarks thermal excitations. Note that the Boltzmann factors is a function of the chiral condensate $\sigma$, therefore the chiral and deconfinement dynamics couples to each other via Eq. (L.Z) and (ㄹ.8). This effect has been investigated in the strong coupling limit case [[12], and now we have the NLO corrections via Eq. ([2.4). Instead of integrating out the temporal link variables, we replace the Polyakov loop with its constant mean-field value, $\left(l_{p, \mathbf{x}}, \bar{l}_{p, \mathbf{x}}\right) \rightarrow\left(l_{p}, \bar{l}_{p}\right)$. Finally, we obtain the effective potential as a function of the auxiliary fields $\Phi=\left(\sigma, \varphi_{\tau, s}, \omega_{\tau}, l_{p}, \bar{l}_{p}\right)$, temperature $T$, and quark chemical potential $\mu$,

$$
\begin{aligned}
\mathscr{F}_{\text {eff }}(\Phi ; T, \mu)= & \left(\frac{d}{4 N_{c}}+\beta_{s} \varphi_{s}\right) \sigma^{2}+\frac{\beta_{s} \varphi_{s}^{2}}{2}+\frac{\beta_{\tau}}{2}\left(\varphi_{\tau}^{2}-\omega_{\tau}^{2}\right)-N_{c} \log \sqrt{Z_{+} Z_{-}} \\
& -N_{c} E_{q}-T\left(\log \mathscr{D}_{q}(T, \mu)+\log \mathscr{D}_{\bar{q}}(T, \mu)\right) \\
& -2 T N_{c}^{2}\left(\frac{1}{g^{2} N_{c}}\right)^{1 / T} \bar{l}_{p} l_{p}-T \log \mathscr{M}_{\text {Haar }}\left(l_{p}, \bar{l}_{p}\right) .
\end{aligned}
$$

The equilibrium is determined by imposing stationary conditions on the effective potential, $\partial \mathscr{F}_{\text {eff }} / \partial \Phi=$

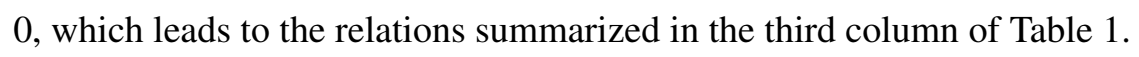
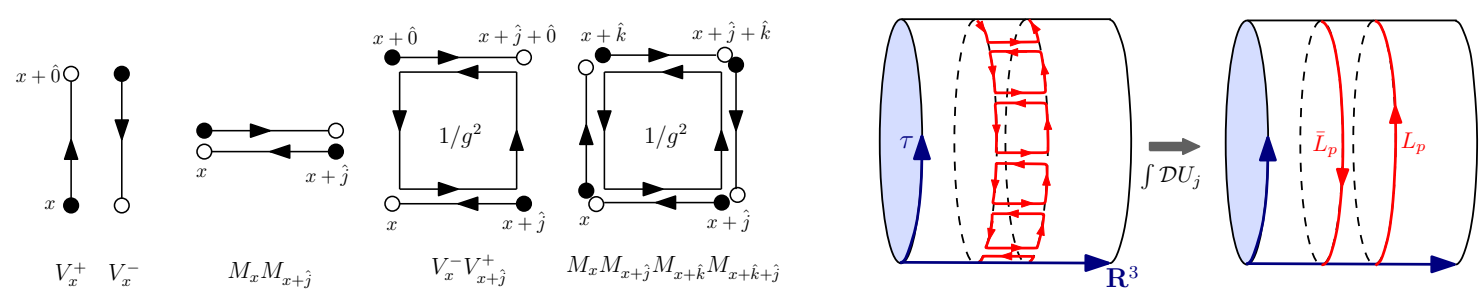

Figure 1: Left: The strong coupling limit and $1 / g^{2}$ effects to appear within the leading order of $1 / d$ expansion. Open circles, filled circles, and arrows show $\chi, \bar{\chi}$, and $U_{v}$, respectively. Right: Schematic figure of the pure gluonic Polyakov loop in the strong coupling expansion. 
Table 1: The auxiliary fields and their stationary values. Here, $\varphi_{0}=N_{c}-\sqrt{Z_{+} Z_{-}} \tilde{m}_{q}+\beta_{\tau} \omega_{\tau}^{2}$.

\begin{tabular}{c|c|c}
\hline Aux. Fields & Mean Fields & Stationary Values \\
\hline$\sigma$ & $\langle-M\rangle$ & $-\partial \mathscr{V}_{q} / \partial\left(\sqrt{Z_{+} Z_{-}} \tilde{m}_{q}\right)$ \\
$\varphi_{s}$ & $\langle M M\rangle$ & $\sigma^{2}$ \\
$\varphi_{\tau}$ & $-\left\langle\left(V^{+}-V^{-}\right) / 2\right\rangle$ & $2 \varphi_{0} /\left(1+\sqrt{1+4 \beta_{\tau} \varphi_{0}}\right)$ \\
$\omega_{\tau}$ & $-\left\langle\left(V^{+}+V^{-}\right) / 2\right\rangle$ & $-\partial \mathscr{V}_{q} / \partial \tilde{\mu}=\rho_{q}$ \\
\hline
\end{tabular}

\section{Results}

We will concentrate on the $\beta=4.0$ results in the followings. In the left panel of Fig. \, we show the phase diagram in the chiral limit. In the low $T$ and large $\mu$ region, we find the critical end point (CEP), and observe a partially restored (PCR) matter around the CEP. The details of PCR have been explained in our previous paper $[\square]$. In this proceedings, we concentrate on the relation between the chiral and deconfinement crossovers. In the right panel of the Fig. [■, the equilibrium value of the Polyakov loop $l_{p}$ is depicted as a function of $T a$ and $\mu a$ in the same condition as the left panel. We find the rapid variation of $l_{p}$ around the chiral transition line. Thus the deconfinement and chiral dynamics are strongly correlated except zero temperature cases.

Let us go along with the $\mu a=0.5$ (dashed blue) line in the right panel of the Fig. ㅁ. The derivative of the Polyakov loop in terms of $T$ on this line is shown in the left panel of Fig. [1]. In the chiral limit ( $m_{0} \rightarrow 0$, left panel), we find two peaks "P" and "Q", and the former corresponds to the steepest point on the $\mu a=0.5$ line in the right panel of Fig. ( (Here, "P" and "Q" in the Fig. [] correspond to those in the Fig. (1). Near the first peak "P", the Polyakov loop is force to change its value to minimize the effective potential due to the drastic variation of the chiral condensate. In this meaning, the peak "P" can be interpreted as the Chiral Induced Deconfinement Crossover. When the current quark mass $m_{0}$ becomes large, the $Z_{N_{c}}$ center symmetry becomes dominant. The right panel in the Fig. B] corresponds to such a situation $\left(m_{0} a=1.0\right)$. We find that the second peak "Q" grows up, and the "P" disappears. This indicates that the second peak is the $Z_{N_{c}}$ Induced Deconfinement Crossover.

It is interesting that the $Z_{N_{c}}$ nature still survives in the chiral limit, and its $\mu$ dependence is almost negligible. Hence the chiral and $Z_{N_{c}}$ dynamics start to separate with increasing $\mu$. This is the mechanism of the two sequential deconfinement crossovers. We note that the coincidence of the chiral and $Z_{N_{c}}$ at " $\mu=0$ " is still mysterious, and to be investigated in future.

\section{Summary}

We have investigated the chiral and deconfinement crossovers at finite temperature $(T)$ and chemical potential $(\mu)$ based on the strong coupling expansion in the lattice QCD with one species of the staggered fermion. We have considered the next-to-leading order (NLO) effects in the strong coupling expansion with the leading pure gluonic contributions to the Polyakov loops. We have concentrate on the leading order of the $1 / d$ expansion, and derived the analytic expression of the effective potential in the framework of the mean-field approximation. We have replaced the 

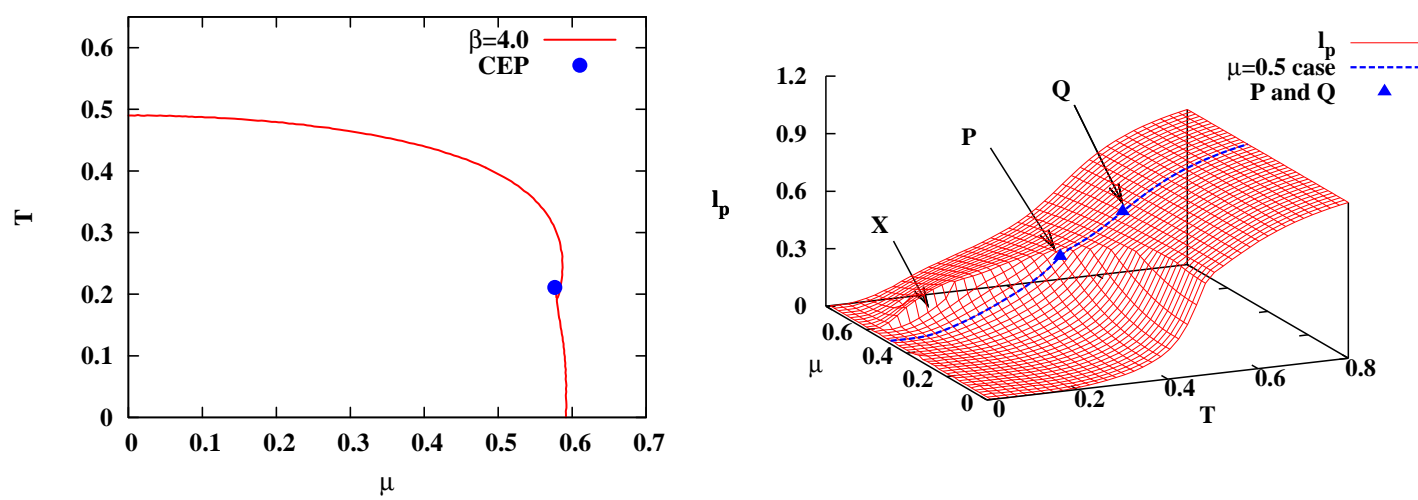

Figure 2: Left: The phase diagram in the chiral limit at $\beta=4.0$. Right: The equilibrium value of the Polyakov loop as a function of $T$ and $\mu$ in the chiral limit at $\beta=4.0$.
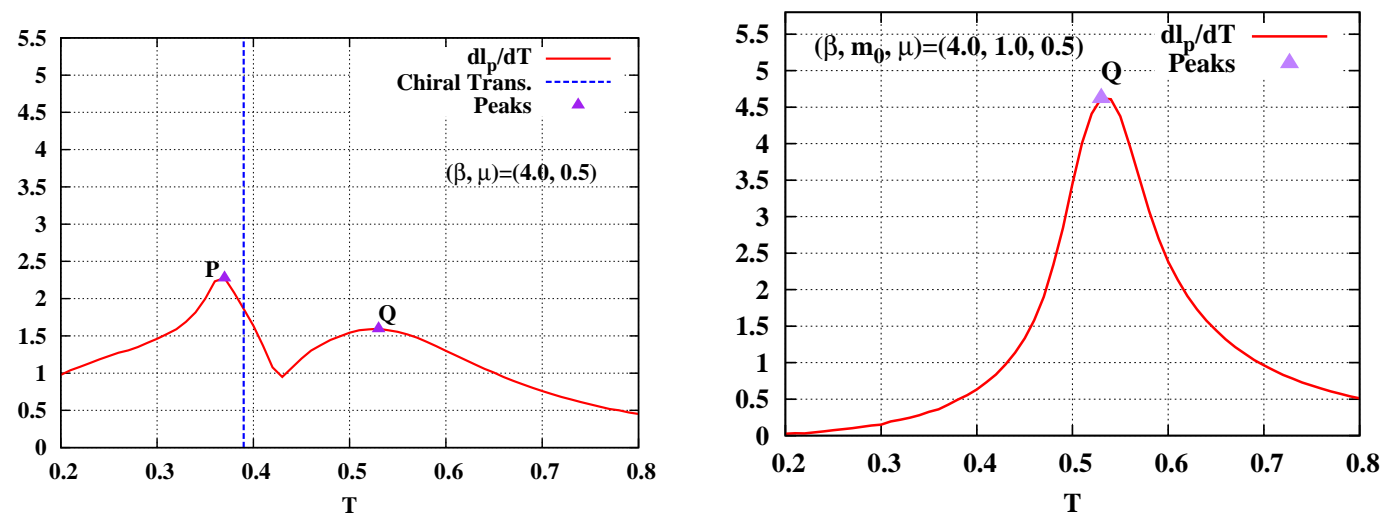

Figure 3: The derivative of the Polyakov loop in terms of $T$ at $\mu=0.5$ in the lattice unit. The left and right panels show the chiral limit and heavy mass $\left(m_{0} a=1.0\right)$ cases, respectively. In the left panel, the vertical dashed (blue) line represents chiral transition temperature.

Polyakov loop with its constant mean fields value without integrating the temporal link variable, and the deconfinement dynamics is introduced via the Haar measure of temporal link integral.

At a fixed large $\mu$, we have observed sequential two deconfinement crossovers i.e. the rapid variations of the Polyakov loops as $T$ increases. One of them results from the strong correlation between the chiral and deconfinement dynamics. In other word, the Polyakov loop is force to change its value to minimize the effective potential due to the drastic variation of the chiral condensate. In this meaning, we have found the Chiral Induced Deconfinement Crossover. When the current quark mass $m_{0}$ becomes large, this crossover disappears, and the another crossover grows up. Thus we have also observed the $Z_{N_{c}}$ Induced Deconfinement Crossover. In the chiral limit, the former becomes stronger, and the latter becomes weaker. The point is that the latter still exists in the chiral limit. Hence two crossovers co-exists there.

There are several studies to be performed in future. Firstly, it has been known that the temporal link integral can be analytically performed, and then the fluctuation effects appear as the 
combination of the modified Bessel function and the difference of Polyakov loops in the effective potential [Q] . It should be confirmed that the obtained results in this work is independent of approximation schemes. Secondly, the chiral and Polyakov loop susceptibilities would be informative to investigate the relation between the chiral and deconfinement dynamics. Thirdly, the exact evaluation in each order of the strong coupling expansion is required beyond the $1 / d$ expansion and the mean-field approximation. This could be achieved by extending the Monomer-Dimer-Polymer formulation [6, [3] to include the NLO effects. And finally, it would be interesting to introduce the advanced formulation of the pure gluonic effects for the Polyakov loops which is recently investigated in Refs. [ए4]].

\section{Acknowledgments}

We thank to Prof. Maria Paola Lombardo for the fruitful discussions. This work was supported in part by Grants-in-Aid for Scientific Research from MEXT and JSPS (Nos. 22-3314), the Yukawa International Program for Quark-hadron Sciences (YIPQS), and by Grants-in-Aid for the global COE program "The Next Generation of Physics, Spun from Universality and Emergence" from MEXT.

\section{References}

[1] For a recent review, see B. Müller and J. L. Nagle, Ann. Rev. Nucl. Part. Sci. 56, (2006) 93.

[2] The review of the pioneering works for the strong coupling expansion is found in the text book, I. Montvay and G. Münster, "Quantum Fields on a Lattice," Cambridge University Press, 1994.

[3] Y. Nishida, K. Fukushima and T. Hatsuda, Phys. Rept. 398 (2004), 281; K. Fukushima, Prog. Theor. Phys. Suppl. 153 (2004), 204; Y. Nishida, Phys. Rev. D 69 (2004), 094501.

[4] V. Azcoiti, G. Di Carlo, A. Galante and V. Laliena, J. High Energy Phys. 09 (2003), 014.

[5] N. Kawamoto, K. Miura, A. Ohnishi and T. Ohnuma, Phys. Rev. D 75 (2007), 014502.

[6] P. de Forcrand and M. Fromm, Phys. Rev. Lett. 104 (2010) 112005.

[7] K. Miura, T. Z. Nakano, A. Ohnishi and N. Kawamoto, Phys. Rev. D 80 (2009) 074034; K. Miura, T. Z Nakano and A. Ohnishi, Prog. Theor. Phys. 122 (2009), 1045.

[8] T. Z. Nakano, K. Miura and A. Ohnishi, Prog. Theor. Phys. 123 (2010) 825.

[9] T. Z. Nakano, K. Miura and A. Ohnishi, arXiv:1009.1518 [hep-lat]; also appear in this proceedings.

[10] H. Kluberg-Stern, A. Morel and B. Petersson, Nucl. Phys. B 215 (1983), 527.

[11] P. H. Damgaard, N. Kawamoto and K. Shigemoto, Nucl. Phys. B 264 (1986), 1.

[12] E. M. Ilgenfritz and J. Kripfganz, Z. Phys. C 29 (1985), 79; A. Gocksch and M. Ogilvie, Phys. Rev. D 31 (1985), 877; K. Fukushima, Phys. Rev. D 68 (2003), 045004.

[13] F. Karsch and K. H. Mutter, Nucl. Phys. B 313, (1989), 541.

[14] J. Langelage and O. Philipsen, JHEP 1004 (2010) 055; JHEP 1001 (2010) 089; J. Langelage, G. Munster and O. Philipsen, JHEP 0807 (2008) 036. 